\title{
Pathologic characteristics of gastric cancer in the elderly: a retrospective study of 994 surgical patients
}

\author{
Tomio Arai ${ }^{1}$, Yukiyoshi Esaki ${ }^{2}$, Naoko Inoshita ${ }^{3}$, Motoji SAwabe $^{1}$, Ichiro Kasahara ${ }^{1}$, Kojiro Kuroiwa $^{4}$, \\ NaOKo Honma ${ }^{5}$, and KaiYo TaKubo \\ ${ }^{1}$ Department of Pathology, Tokyo Metropolitan Geriatric Medical Center, 35-2 Sakae-cho, Itabashi-ku, Tokyo 173-0015, Japan \\ ${ }^{2}$ Department of Pathology, Sayama Hospital, Sayama, Japan \\ ${ }^{3}$ Department of Pathology, Cancer Institute, Tokyo, Japan \\ ${ }^{4}$ Department of Surgery, Tokyo Metropolitan Geriatric Medical Center, Tokyo, Japan \\ ${ }^{5}$ Human Tissue Research Group, Tokyo Metropolitan Institute of Gerontology, Tokyo, Japan
}

\begin{abstract}
Background. The clinicopathologic features of gastric carcinoma in elderly people have been reported previously. The present study examined the patterns and distribution of gastric carcinomas in the elderly, especially in patients aged 85 and older.

Methods. A retrospective study of 994 consecutive Japanese patients aged 65 years or older was performed. In this group, a total of 1147 lesions were analyzed. Pathological findings in the very old group (older than 85 years; $n=126$ ) were compared with those in younger groups (65-74 years [young-old group]; $n=356)$ and (75-84 years [middle-old group]; $n=512)$.

Results. While the male-to-female ratio significantly decreased with advancing age, the relative odds of gastric cancer in men were higher than those in women in all age groups. In the very old group, cancer of the lower third of the stomach tended to increase with advancing age, and accounted for $43.7 \%$ of cases. In the population overall, differentiated-type adenocarcinoma accounted for $\mathbf{8 9 . 6} \%$ in the early cancers and $50.3 \%$ in the advanced cancers. The proportion of cases involving differentiated-type carcinoma significantly increased with advancing age in early cancer and female advanced cancer cases, whereas no significant change was found in male advanced-cancer patients. In the very old group, lymph node metastasis was found in $\mathbf{5 . 4 \%}$ of early cancers and $\mathbf{7 2 . 7} \%$ in advanced cancers. Multiple cancers significantly increased with advancing age $(P<0.05 ; 10.7 \%$ in the younger-old group, $12.7 \%$ in the middle-old group, and $19.0 \%$ in the very old group).

Conclusion. These results indicate that, in the very old group, gastric cancers showed a distal shift with predominantly differentiated-type carcinoma in the early stages and increased undifferentiated-type carcinomas in advanced stages. These results suggest increased histologic diversity with tumor growth. These findings have important implications for the screening and diagnosis of gastric cancer in the elderly.
\end{abstract}

Key words Carcinoma $\cdot$ Stomach $\cdot$ Elderly $\cdot$ Differentiatedtype carcinoma

\section{Introduction}

The incidence of gastric cancer in elderly people has recently been increasing in Japan due to the extension of the life span in the general population. This has been an ongoing trend despite an overall decrease in the incidence of gastric cancer in the country. It is estimated that gastric cancer in patients aged 65 years or older accounts for approximately $70 \%$ of total gastric cancers [1]. Moreover, age-adjusted death rates for stomach cancer have increased exponentially with aging [2]. This evidence indicates that the relationship of aging to cancer in elderly patients is of great importance.

The characteristic features of gastric carcinoma in elderly people have been reported by several investigators [3-9]. Previous studies have shown that gastric cancer involving the lower third of the stomach and histopathologically well-differentiated adenocarcinoma was significantly more prevalent in elderly patients $[6,8$, 10]. It is also reported that the frequency of advanced cancer in the elderly is higher than in young people [7], and multiple and metastatic cancers are more common in aged people [5,11]. However, only a few reports, using a relatively small number of cases, have examined the clinicopathologic features of gastric carcinoma in very old people. The present study focused on this point by examining the patterns and distribution of gastric carcinomas in the elderly, especially in patients aged 85 and older.

\section{Patients and methods}

A total of 994 consecutive patients aged 65 years or older, with a total of 1147 carcinomas of the stomach, 
were selected from patients at the Tokyo Metropolitan Geriatric Medical Center between January 1984 and December 2003. Of the 1147 carcinomas, 1135 lesions (982 patients) were surgically resected, and 12 lesions (12 patients; 11 intramucosal cancers and 1 cancer with submucosal invasion) were endoscopically resected. The tumors were histopathologically examined as part of the routine procedure. Resectability rates of gastric carcinoma in the hospital between May 1986 and December 1994 were $79.1 \%$ (155/196) in the young-old group (see below for age range), 75.7\% (240/317) in the middle-old group, and 58.3\% (56/96) in the very old group. In elderly patients who did not undergo resection of the tumor, some were found to have unresectable disease because of metastasis. These metastases included liver metastasis, peritoneal dissemination, and distant lymph node involvement. Other patients who did not undergo resection had severe complications, such as chronic heart failure, myocardial infarction, diabetes, and aspiration pneumonia.

We reviewed pathological protocols and microscopic slides in each case. We also analyzed records for age, sex, location of the tumor, histological type, depth of invasion, lymph node metastasis, and the presence of two or more gastric carcinomas. The anatomical site of gastric tumors was classified based on surgical records and pathological reports. Histological types were described on the basis of the Japanese classification of gastric carcinoma [12].

All patients were divided into three age groups: young-old group (65-74 years old; 356 patients with 413 lesions), middle-old group (75-84 years old; 512 patients with 583 lesions), and a very old group (85 years and older; 126 patients with 151 lesions). We compared the parameters in the very old group with those in the younger groups. Furthermore, we compared the data of the earlier decade (1984-1993) with those of the following decade (1994-2003) due to the long observation period.

Differences among groups were tested for statistical significance using a $\chi^{2}$ test. A $P$ value less than 0.05 (twosided) was considered significant. Because the greater longevity of females may produce an apparent increase in the number of female patients with gastric cancer, the relative odds of gastric carcinoma in both sexes were calculated and compared with each other. The relative odds for males were calculated according to the following formula: $\mathrm{p}\left(1-\mathrm{p}_{0}\right) / \mathrm{p}_{0}(1-\mathrm{p}) ; \mathrm{p}=$ ratio of male patients with gastric cancer in each age group, and $\mathrm{p}_{0}=$ ratio of males in the general Japanese population in the same age group [13]. Female odds were calculated in the same way. The numbers of individuals in each age group in the general population during the period from 1985 to 2000 were obtained from the Vital statistics of Japan [1].

\section{Results}

The percentages of men decreased with age $(66.1 \%$ in the young-old group, $53.5 \%$ in the middle-old group, and $50.8 \%$ in the very old group). The young-old group consisted of more men than women $(P=0.0003)$. The relative odds of having gastric cancers in men were higher than those in women in all age groups (Table 1). Site distribution of gastric cancers is shown in Table 1. Cancer of the lower third of the stomach in the very old group accounted for $43.7 \%$. This was a slightly higher rate than in the younger groups. The percentage of cancer of the lower third of the stomach in women $(41.5 \%)$ was higher than that in men $(36.5 \%)$. Earlyto-advanced cancer ratios were approximately equal in all age groups. Elevated-type cancer increased with age in both early and advanced cancers (Table 1). On the other hand, the percentage of type 4 cancer (diffuse-infiltrative type) in women $(17.7 \%)$ was higher than that found in men $(13.7 \%)$. In the very old group, differentiated-type adenocarcinoma accounted for $89.6 \%$ of the early cancers and $50.3 \%$ of the advanced cancers (Table 1). The proportion of differentiated-type carcinoma increased with advancing age in early cancer and female advanced cancers, whereas no significant change was found in male advanced cancer (Fig. 1). The proportion of differentiated-type carcinoma in men was

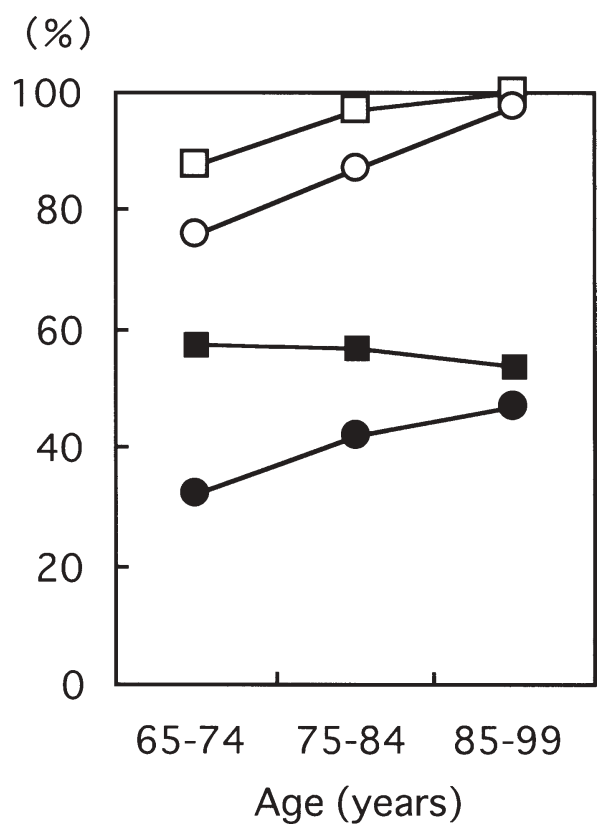

Fig. 1. Proportions of differentiated-type carcinoma in early and advanced cancers. Note increased proportion of differentiated-type carcinoma with advancing age in early and female advanced cancer, but there was no significant change in male advanced cancer. Open squares, male early cancer; open circles, female early cancer; closed squares, male advanced cancer; closed circles, female advanced cancer 
Table 1. Characteristics of gastric cancer in 994 elderly patients

\begin{tabular}{|c|c|c|c|c|}
\hline \multirow[b]{2}{*}{ No. of lesions } & \multicolumn{3}{|c|}{ Age groups, in years } & \multirow[b]{2}{*}{ All $(n=1147)$} \\
\hline & $65-74(n=413)$ & $75-84(n=583)$ & $85-99(n=151)$ & \\
\hline $\begin{aligned} \text { Male: Female** } & \\
& \text { (Percentages of men) }\end{aligned}$ & $235: 121(66.1)$ & $274: 238(53.5)$ & $64: 62(50.8)$ & $573: 421(57.7)$ \\
\hline \multicolumn{5}{|l|}{ Relative odds of gastric cancer } \\
\hline Male & 2.41 & 1.89 & 112.09 & 1.90 \\
\hline Female & 0.41 & 0.53 & 10.48 & 0.53 \\
\hline \multicolumn{5}{|l|}{ Tumor location } \\
\hline Upper third & $62(15.0)$ & $90(15.4)$ & $16(10.6)$ & $168(14.6)$ \\
\hline Middle third & $172(41.7)$ & $258(44.3)$ & $61(40.4)$ & $491(42.8)$ \\
\hline Lower third & $161(39.0)$ & $216(37.0)$ & $66(43.7)$ & $443(38.6)$ \\
\hline Whole & $7(1.7)$ & $14(2.4)$ & $6(4.0)$ & $27(2.4)$ \\
\hline Remnant stomach & $11(2.7)$ & $5(0.9)$ & $2(1.3)$ & 18 (1.6) \\
\hline Early: Advanced cancers & $203: 210$ & $272: 311$ & $74: 77$ & $549: 598$ \\
\hline \multicolumn{5}{|l|}{ Gross features } \\
\hline \multicolumn{5}{|l|}{ Early cancer } \\
\hline I & $22(10.8)$ & $36(13.2)$ & $12(16.2)$ & $70(12.8)$ \\
\hline IIa & $56(27.6)$ & $92(33.8)$ & $24(32.4)$ & $172(31.3)$ \\
\hline IIb & $19(9.4)$ & 31 (11.4) & $6(8.1)$ & $56(10.2)$ \\
\hline IIc & $105(51.7)$ & $111(40.8)$ & $32(43.3)$ & $248(45.2)$ \\
\hline III & $1(0.5)$ & $2(0.8)$ & 0 & $3(0.6)$ \\
\hline \multicolumn{5}{|l|}{ Advanced cancer } \\
\hline 1 & $11(5.2)$ & $21(6.8)$ & $7(9.1)$ & $39(6.5)$ \\
\hline 2 & $71(33.8)$ & $109(35.0)$ & $25(32.4)$ & $205(34.3)$ \\
\hline 3 & $68(32.4)$ & $99(31.8)$ & $24(31.2)$ & $191(31.9)$ \\
\hline 4 & $35(16.7)$ & $42(13.5)$ & $14(18.2)$ & $91(15.2)$ \\
\hline 5 & 25 (11.9) & $40(12.9)$ & $7(9.1)$ & $72(12.1)$ \\
\hline Cancer $\geq 5 \mathrm{~cm}$ & $187(45.3)$ & $291(49.9)$ & $72(47.7)$ & $550(48.0)$ \\
\hline \multicolumn{5}{|l|}{ Differentiated-type carcinoma } \\
\hline Early cancer** & $169 / 203(83.3)$ & 250/272 (91.9) & 73/74 (98.6) & $492 / 549(89.6)$ \\
\hline Advanced cancer & $106 / 210(50.5)$ & $156 / 311(50.2)$ & $39 / 77(50.6)$ & $301 / 598(50.3)$ \\
\hline \multicolumn{5}{|l|}{ Lymph node metastasis } \\
\hline Early cancer & $14 / 203(6.9)$ & 19/272 (7.0) & 4/74 (5.4) & $37 / 549(6.7)$ \\
\hline Advanced cancer & $153 / 210(72.9)$ & 237/311 (76.2) & $56 / 77(72.7)$ & $446 / 598(74.6)$ \\
\hline $\begin{array}{l}\text { Two or more cancers in the } \\
\text { stomach* }\end{array}$ & $38 / 356(10.7)$ & $65 / 512(12.7)$ & $24 / 126(19.0)$ & $127 / 994(12.8)$ \\
\hline
\end{tabular}

$* P<0.05 ; * * P<0.01$

Values are numbers, with percentages in parentheses

higher than that in women in all age groups (Fig. 1). In the very old group, the proportion showing lymph node metastasis was $5.4 \%$ in early cancer and $72.7 \%$ in advanced cancers (Table 1 ). In early cancer, lymph node metastasis was more common in men than in women in all age groups (Fig. 2). In women, the rate of lymph node metastasis decreased with advancing age, whereas in men metastasis peaked in the middle-old group (Fig. 2). Multiple gastric cancers increased with advancing age $(P=0.037 ; 10.7 \%$ in the young-old group, $12.7 \%$ in the middle-old group, and $19.0 \%$ in the very old group). Multiplicity of gastric cancers in men was more frequent than in women (Fig. 3).

The proportion of lower-third gastric cancer in the most recent decade was higher than that in the previous decade (Fig. 4), even though age and sex distribution was not different between the two periods. Other than a decreasing trend in carcinoma of the upper third of stomach in the very old group, there were no significant changes between the two periods. Gross features, histology, and the early-to-advanced cancer ratio showed no significant difference between the two time periods. In the middle-old and very old groups, multiplicity of gastric cancer in the recent decade (1994-2003) was less common than in the previous decade (1984-1993), whereas in the young-old group there was a slight increase in the multiplicity of gastric cancer (Fig. 5) in the most recent decade.

\section{Discussion}

In the present study, we analyzed gastric cancers in elderly patients, from the standpoint of aging. We found that, in the very old group, tumors occurred predominantly in the lower third of the stomach; differentiated- 


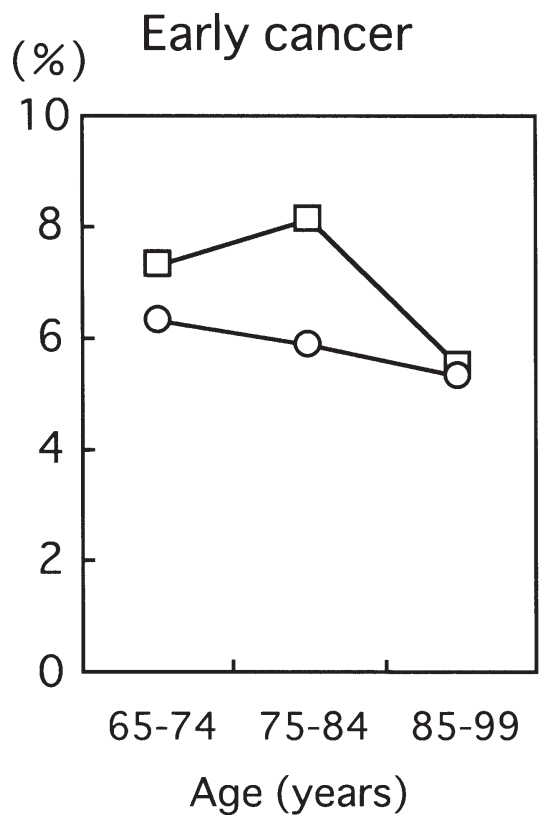

(\%)

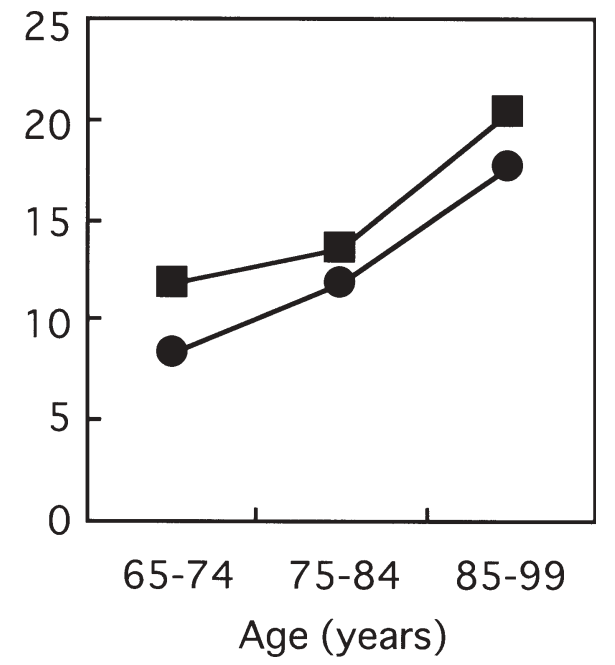

Fig. 3. Multiplicity of gastric cancers. Multiple gastric cancers increased with advancing age in both sexes. Closed squares, male; closed circles, female

type carcinoma was more common in early cancer; and multiple cancers increased with advancing age. Moreover, we found sex differences in histological type, lymph node metastasis, multiple cancers, and time trends in tumor location.

It is generally documented that gastric cancer in elderly patients is predominantly localized to the lower third of the stomach [6-9,14-18]. Previous reports demonstrated that the proportion (range, $42 \%-63 \%$ ) of carcinomas in the lower third of the stomach in the elderly was higher than the proportion (range, 31\%$44 \%)$ in the young $[3,6-9,14-19]$. Our data confirmed that, in patients aged 85 years or older, cancers were situated more often in the lower third of the stomach compared with the younger age groups. Thus, it is conceivable that the risk of developing carcinoma in the lower third of the stomach increases with advancing age. This trend held true even in individuals 85 years or older. On the other hand, there was no clear trend of risk patterns involving carcinoma of the upper third of the stomach. Some investigators have reported that gastric cancer involving the upper third of the stomach in the elderly was more common than in younger groups [6,7,15-17,19], whereas others found no significant difference or lower prevalence in the elderly group $[3,8,14,18]$.

Several reports have indicated that differentiatedtype or intestinal-type carcinomas are more common in older patients than in younger patients [3,5-8,14$16,18,19]$. Our study demonstrated that this tendency was clear, especially in early-stage cancer. We also found that the proportion of differentiated-type carcinoma increased with advancing age, reaching a level greater than $95 \%$. These findings confirm evidence presented in our previous report [8]. Our findings demonstrated that, in cases of advanced gastric cancer, the proportion of differentiated-type carcinomas was almost equal to that of undifferentiated-type carcinoma. These results suggest that gastric cancer in the elderly demonstrates increased histological diversity during growth [8]. 


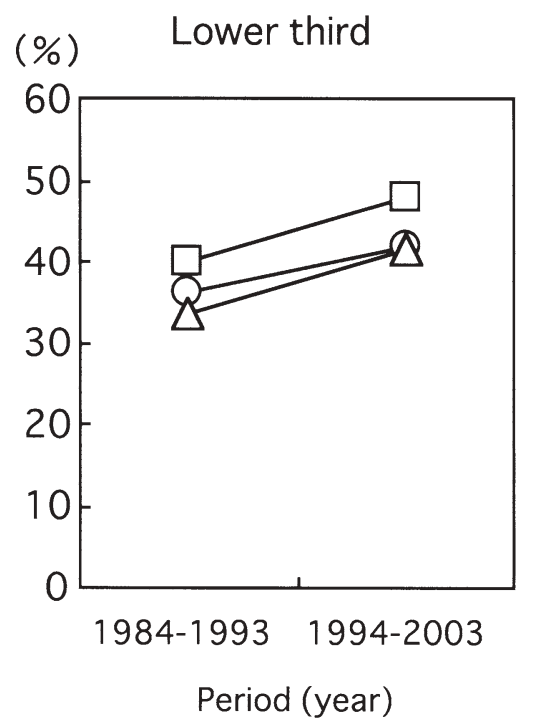

(\%)

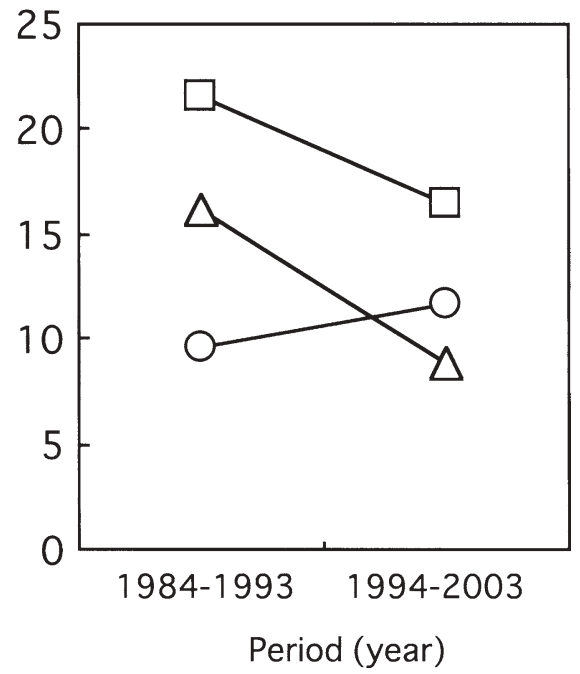

Fig. 5. Time trends in the proportion of multiple gastric cancers over 20 years. Note slight increasing trend in the youngold group and marked decreasing trend in the older groups. Open circles, young-old group (65-74 years); open triangles, middle-old group (75-84 years); open squares, very old group (85-99 years)

Previous reports have shown no significant difference in the incidence of lymph node metastasis between young and elderly patients with gastric cancer [3,57,14-19]. Most of these reports did not take the clinical or pathological stage of the disease into account, and the results could not be simply compared with each other. However, our findings, and a few other reports $[6,14]$, suggest that the incidence of lymph node metastasis in the elderly is lower than that in younger patients in cases of early gastric cancer. Moreover, examination of autopsy cases of fatal gastric cancer showed that younger patients had a higher incidence of lymph node metastasis [20]. Thus, in the very old group, cancer tissue may metastasize less often to the regional lymph node compared with younger patients.

In general, multiple cancers are more common problems in elderly patients compared to younger populations [2]. The present study provided evidence that approximately $13 \%$ of our total population of elderly patients with gastric cancer had two or more cancers within the stomach, which is consistent with previous reports $[5,11,15,21]$. Although the incidence of multiple colorectal cancers was not significantly different among the age groups $[13,22,23]$, multiple gastric cancers increased with advancing age $[15,21,24]$.

Sex differences were recognized in site distribution, histologic type, and multiplicity. Our findings demonstrated a higher incidence of gastric cancer affecting the lower third of the stomach or whole stomach in females. Both differentiated-type and multiple gastric cancer sites were more common in men than in women in all age groups. In other studies, undifferentiated-type carcinoma was found more frequently in young women, whereas differentiated-type carcinoma primarily affected elderly men. Generally, undifferentiated-type carcinoma involves the whole stomach, which results in "linitis plasitca". Most multiple gastric cancers are composed of differentiated-type carcinoma [11]. Thus, sex differences in histologic type may play an important role in tumor location and multiplicity. Sex differences in histologic type may be due to intrinsic and environmental factors, such as sex hormones, bile acid, fatty acid, H. pylori infection, and alcohol intake. However, this issue remains unclear.

The anatomical site distribution of gastric cancer differed in time trends between the very old group ( 85 years or older) and the younger groups (65-84 years 
old). Our findings demonstrated a decreasing trend of carcinoma of the upper third of the stomach in the very old group and no significant change in the younger groups. This finding suggests that an age-related decreasing trend of carcinoma of the upper third of the stomach is no longer occurring in the younger groups. These trends have been found in the 1980s and 1990s in the United States and Europe [25-29]. These studies provide support for the idea that the incidence of tumors located in the distal part of the stomach has declined, whereas cancers of the proximal stomach have been rapidly increasing. Age-period-cohort analysis suggested that the proportions of the locations of gastric carcinomas may, to some extent, reflect a birthcohort phenomenon [25]. This birth-cohort phenomenon influences the time trend of both cardia and distal gastric carcinomas and suggests that the incidence of carcinoma of the proximal stomach will increase in the future because the prevalence of lower esophageal adenocarcinoma and cardia carcinoma is currently increasing worldwide $[25,28]$.

In conclusion, we found a distal shift in cancer location and increased multiplicity of gastric cancer with advancing age. We also found that differentiated-type carcinoma was more common in early disease stages and undifferentiated-type carcinoma was more common in advanced disease stages, which may indicate increased histologic diversity with tumor growth. These findings have important implications for the screening and diagnosis of gastric cancer in the elderly.

Acknowledgments We thank the staff at the Department of Pathology, Tokyo Metropolitan Geriatric Medical Center for their excellent technical assistance. The study was supported, in part, by Novartis Foundation for Gerontological Research 2003.

\section{References}

1. Statistics and Information Department, Minister's Secretriat. Vital statistics of Japan. Tokyo: Ministry of Health and Welfare of Japan; 2000.

2. Arai T, Takubo K, Esaki Y. Carcinogenesis and aging. Turk J Cancer 1997;27:131-8.

3. Habu H, Endo M. Gastric cancer in elderly patients - results of surgical treatment. Hepatogastroenterology 1989;36:71-4.

4. Edelman DS, Russin DJ, Wallack MK. Gastric cancer in the elderly. Am Surg 1987;53:170-3.

5. Kitamura K, Yamaguchi T, Taniguchi H, Hagiwara A, Yamane T, Sawai K, et al. Clinicopathological characteristics of gastric cancer in the elderly. Br J Cancer 1996;73:798-802.

6. Ishigami S, Natsugoe S, Saihara T, Hokita S, Tokushige M, Watanabe T, et al. Clinical and pathologic features of early gastric cancer in elderly patients. Hepatogastroenterology 1997;44:1164-8.

7. Hanazaki K, Wakabayashi M, Sodeyama H, Miyazawa M, Yokoyama S, Sode Y, et al. Surgery for gastric cancer in patients older than 80 years of age. Hepatogastroenterology 1998;45:26875 .
8. Inoshita N, Yanagisawa A, Arai T, Kitagawa T, Hirokawa K, Kato Y. Pathological characteristics of gastric carcinomas in the very old. Jpn J Cancer Res 1998;89:1087-92.

9. Medina-Franco H, Heslin MJ, Cortes-Gonzalez R. Clinicopathological characteristics of gastric carcinoma in young and elderly patients: a comparative study. Ann Surg Oncol 2000;7:515-9.

10. Nakajima T, Akiyama Y, Shiraishi J, Arai T, Yanagisawa Y, Ara $\mathrm{M}$, et al. Age-related hypermethylation of the hMLH1 promoter in gastric cancers. Int J Cancer 2001;94:208-11.

11. Esaki Y, Hirokawa K, Yamashiro M. Multiple gastric cancers in the aged with special reference to intramucosal cancers. Cancer 1987;59:560-5.

12. Japanese Gastric Cancer Association. Japanese classification of gastric carcinoma. 2nd English Ed. Gastric Cancer 1998;1:10-24.

13. Arai T, Takubo K, Sawabe M, Esaki Y. Pathologic characteristics of colorectal cancer in the elderly: a retrospective study of 947 surgical cases. J Clin Gastroenterol 2000;31:67-72.

14. Fujimoto S, Takahashi M, Ohkubo H, Mutou T, Kure M, Masaoka H, et al. Comparative clinicopathologic features of early gastric cancer in young and older patients. Surgery 1994;115:51620.

15. Maehara Y, Emi Y, Tomisaki S, Oshiro T, Kakeji Y, Ichiyoshi Y, et al. Age-related characteristics of gastric carcinoma in young and elderly patients. Cancer 1996;77:1774-80.

16. Wang JY, Hsieh JS, Huang CJ, Huang YS, Huang TJ. Clinicopathologic study of advanced gastric cancer without serosal invasion in young and old patients. J Surg Oncol 1996;63:36-40.

17. Kubota H, Kotoh T, Dhar DK, Masunaga R, Tachibana M, Tabara $\mathrm{H}$, et al. Gastric resection in the aged ( $>$ or $=80$ years) with gastric carcinoma: a multivariate analysis of prognostic factors. Aust N Z J Surg 2000;70:254-7.

18. Okuno K, Shigeoka H, Tanaka A, Hirai N, Matsumura E, Yasutomi M. Clinicopathological evaluation of T2-gastric cancer among age groups. Hepatogastroenterology 2000;47:1180-2.

19. Wu CW, Lo SS, Shen KH, Hsieh MC, Lui WY, P'Eng FK. Surgical mortality, survival, and quality of life after resection for gastric cancer in the elderly. World J Surg 2000;24:465-72.

20. Esaki Y, Hirayama R, Hirokawa K. A comparison of patterns of metastasis in gastric cancer by histologic type and age. Cancer 1990;65:2086-90.

21. Mitsudomi T, Watanabe A, Matsusaka T, Fujinaga Y, Fuchigami T, Iwashita A. A clinicopathological study of synchronous multiple gastric cancer. Br J Surg 1989;76:237-40.

22. Kimura T, Iwagaki H, Fuchimoto S, Hizuta A, Orita K. Synchronous colorectal carcinomas. Hepatogastroenterology 1994;41: 409-12.

23. Arai T, Sawabe M, Takubo K, Kanazawa K, Esaki Y. Multiple colorectal cancers in the elderly: a retrospective study of both surgical and autopsy cases. J Gastroenterol 2001;36:748-52.

24. Kitamura K, Yamaguchi T, Okamoto K, Otsuji E, Taniguchi H, Hagiwara A, et al. Clinicopathologic features of synchronous multifocal early gastric cancers. Anticancer Res 1997;17:643-6.

25. Zheng T, Mayne ST, Holford TR, Boyle P, Liu W, Chen Y, et al. The time trend and age-period-cohort effects on incidence of adenocarcinoma of the stomach in Connecticut from 1955-1989. Cancer 1993:72:330-40.

26. Devesa SS, Blot WJ, Fraumeni JF Jr. Changing patterns in the incidence of esophageal and gastric carcinoma in the United States. Cancer 1998;83:2049-53.

27. Hassan HA, Sharma VK, Raufman JP. Changing trends in gastric carcinoma at a university medical center: a 12-year retrospective analysis. J Clin Gastroenterol 2001;32:37-40.

28. Popiela T, Kulig J, Kolodziejczyk P, Sierzega M. Changing patterns of gastric carcinoma over the past two decades in a single institution: clinicopathological findings in 1557 patients. Scand J Gastroenterol 2002;37:561-7.

29. Golematis B, Tzardis P, Hatzikostas P, Papadimitriou K, Haritopoulos N. Changing pattern of distribution of carcinoma of the stomach. Br J Surg 1990;77:63-4. 